\title{
Experimentally Verified 2D Systems Theory based Robust Iterative Learning Control
}

\author{
Lukasz Hladowski, Krzysztof Galkowski, Zhonglun Cai, Eric Rogers, Chris T. Freeman, Paul L. Lewin
}

\begin{abstract}
This paper uses 2D control systems theory to develop robust iterative learning control laws for linear plants with experimental validation on a gantry robot used for 'pick and place' operations commonly found in industries such as food processing. In particular, the stability theory for linear repetitive processes provides the setting for analysis and this allows design to take account of trial-to-trial error convergence, transient response along the trials and robustness. The mechanism for this is the use of a strong form of stability for repetitive processes/2D linear systems known as stability along the pass (or trial) with the added requirement for maintaining this property in the presence of model uncertainty. The resulting design computations are in terms of Linear Matrix Inequalities (LMIs) and the control laws can be implemented without the need to estimate state vector entries.
\end{abstract}

\section{INTRODUCTION}

Iterative learning control (ILC) is a technique for controlling systems operating in a repetitive (or pass-to-pass) mode with the requirement that a reference trajectory $y_{\text {ref }}(t)$ defined over a finite interval $0 \leq t \leq \alpha$ is followed to a high precision. Examples of such systems include robotic manipulators that are required to repeat a given task to high precision, chemical batch processes or, more generally, the class of tracking systems.

Since the original work [1], the general area of ILC has been the subject of intense research effort. An initial source for the literature here are the survey papers [2] and [3]. The analysis of ILC schemes is firmly outside standard (or 1D) control theory, although it is still has a significant role to play in certain cases of practical interest. Such schemes do, however, have a natural 2D systems structure and hence we make use of the links with repetitive processes where information propagation in one of the two directions only occurs over a finite duration.

In ILC, a major objective is to achieve convergence of the trial-to-trial error and often this has been treated as the only objective. In fact, it is possible that enforcing fast convergence could lead to unsatisfactory along the trial performance. Moreover, in many applications there will be uncertainty associated with the model used for design and hence a robust control theory that takes account of this tradeoff is required.

L. Hladowski and K. Galkowski are with the Institute of Control and Computation Engineering, University of Zielona Gora, Poland L.Hladowski@issi.uz.zgora.pl, K.Galkowski@issi.uz.zgora.pl

Z. Cai, E. Rogers, C. T. Freeman and P. L. Lewin are with the School of Electronics and Computer Science, University of Southampton, Southampton SO17 1BJ, UK

This work has been partially supported by the Ministry of Science and Higher Education in Poland under the project N N514 293235.
This paper shows how robust ILC, with due regard to both trial-to-trial error convergence and along the trial performance, can be achieved by extending robust control law design algorithms for discrete linear repetitive processes. The key feature here is the use of the strong concept of stability along the pass (or trial) for these processes in an ILC setting. The analysis results in control law design algorithms that can be computed using Linear Matrix Inequalities (LMIs) and the laws themselves have a well defined physical structure. Finally, representative results from the experimental application of the algorithms developed to a gantry robot facility that executes a pick and place operation commonly found in the process industries are given, where the plant modeling starts from frequency response data.

In this paper the null and identity matrices with the required dimensions are denoted by 0 and $I$ respectively. Also $\Gamma \succ 0$ and $\Gamma \prec 0$ are used to denote symmetric matrices that are positive definite and negative definite, respectively. The symbol $\mathrm{r}(\cdot)$ is used to denote the spectral radius of a given matrix. In particular, if $M$ is a $p \times p$ matrix with eigenvalues $\lambda_{i}, 1 \leq i \leq p$, then $r(M)=\max _{1 \leq i \leq p}\left|\lambda_{i}\right|$. Finally, $*$ is used to denote block entries in symmetric LMIs.

\section{BACKGROUND}

Consider the case when the plant to be controlled can be modeled as a differential linear time-invariant system with state-space model defined (state, input and output matrices respectively) by $\{A, B, C\}$. Suppose also that this model is sampled using a zero-order hold. Then in an ILC setting the discrete dynamics are written as

$$
\begin{aligned}
x_{k}(p+1) & =A x_{k}(p)+B u_{k}(p), \\
y_{k}(p) & =C x_{k}(p), p=0,1, \ldots, \alpha-1,
\end{aligned}
$$

where on trial $k, x_{k}(p) \in \mathbb{R}^{n}$ is the state vector, $y_{k}(p) \in \mathbb{R}^{m}$ is the output vector, $u_{k}(p) \in \mathbb{R}^{p}$ is the vector of control inputs, and the trial length $\alpha<\infty$. If the signal to be tracked is denoted by $y_{r e f}(p)$ then $e_{k}(p)=y_{r e f}(p)-y_{k}(p)$ is the error on trial $k$, and the most basic requirement is to force the error to converge in $k$. In particular, the objective of constructing a sequence of input functions such that the performance is gradually improving with each successive trial can be refined to a convergence condition on the input and error

$$
\lim _{k \rightarrow \infty}\left\|e_{k}\right\|=0, \lim _{k \rightarrow \infty}\left\|u_{k}-u_{\infty}\right\|=0,
$$

where $\|\cdot\|$ is a signal norm in a suitably chosen function space with a norm-based topology. 
It is, however, possible that trial-to-trial error convergence will occur but produce along the trial performance that is far from satisfactory for many practical applications, e.g. a gantry robot whose task is to collect an object from a location, place it on a moving conveyor, and then return for the next one and so on. If, for example, the object has an open top and is filled with liquid, and/or is fragile in nature, then unwanted vibrations during the transfer time could have very detrimental effects. Hence in such cases there is also a need to control the along the trial dynamics and in this paper the method is to use a stronger form of stability theory for linear repetitive processes.

One approach to the analysis of ILC schemes with the potential to address the dynamics in both directions, i.e. trialto-trial and along the trial respectively is to use a $2 \mathrm{D}$ systems setting. For example, in [4] it was shown how trial-to-trial error convergence of linear ILC schemes in the discrete domain could be examined as a stability problem in terms of a 2D discrete linear systems state-space model interpretation of the dynamics. To-date, however, relatively little attention has been directed towards control law design in a 2D systems setting for both trial-to-trial convergence and along the trial dynamics.

Given that the trial length is finite by definition, it follows that ILC fits naturally into the class of so-called repetitive processes [5]. The unique characteristic of such a process is a series of sweeps, termed passes, through a set of dynamics defined over a fixed finite duration known as the pass length. On each pass an output, termed the pass profile, is produced which acts as a forcing function on, and hence contributes to, the dynamics of the next pass profile. This, in turn, leads to the unique control problem that the output sequence of pass profiles generated can contain oscillations that increase in amplitude in the pass-to-pass direction.

Attempts to control these processes using standard systems theory and algorithms fail (except in a few very restrictive special cases) precisely because such an approach ignores their inherent 2D systems structure, i.e. information propagation occurs from pass-to-pass ( $k$ direction) and along a given pass ( $t$ direction) and also the initial conditions are reset before the start of each new pass. To remove these deficiencies, a rigorous stability theory has been developed [5] based on an abstract model of the dynamics in a Banach space setting that includes a very large class of processes with linear dynamics and a constant pass length as special cases, including those described by (2) below. In terms of their dynamics, it is the pass-to-pass coupling (noting again their unique feature) which is critical. This is of the form $y_{k+1}=L_{\alpha} y_{k}$, where $y_{k} \in E_{\alpha}\left(E_{\alpha}\right.$ a Banach space with norm $\left.\|\cdot\|\right)$ and $L_{\alpha}$ is a bounded linear operator mapping $E_{\alpha}$ into itself.

Consider now discrete linear repetitive processes described by the following state-space model over $p=0,1, \ldots, \alpha-$ $1, k \geq 0$,

$$
\begin{aligned}
x_{k+1}(p+1) & =\hat{A} x_{k+1}(p)+\hat{B} u_{k+1}(p)+\hat{B}_{0} y_{k}(p), \\
y_{k+1}(p) & =\hat{C} x_{k+1}(p)+\hat{D} u_{k+1}(p)+\hat{D}_{0} y_{k}(p)
\end{aligned}
$$

where on pass, or trial in an ILC setting, $k, x_{k}(p) \in \mathbb{R}^{n}$ is the state vector, $y_{k}(p) \in \mathbb{R}^{m}$ is the pass profile vector and $u_{k}(p) \in \mathbb{R}^{r}$ is the control input vector. To complete the process description, it is necessary to specify the initial, or boundary, conditions, i.e. the state initial vector on each pass and the initial pass profile. Here these are taken to be zero. In the next section, it is shown how a repetitive process setting can be used to analyze ILC schemes and, in particular, how the stability theory of these processes can be employed to develop algorithms for control law design for trial-to-trial error convergence and along the trial performance.

The stability theory [5] for linear repetitive processes consists of two distinct concepts. Asymptotic stability for (1) holds if, and only if, $\mathrm{r}\left(\hat{D}_{0}\right)<1$. Also if this property holds and the control input sequence applied $\left\{u_{k}\right\}_{k}$ converges strongly to $u_{\infty}$ as $k \rightarrow \infty$ then the resulting output pass profile sequence $\left\{y_{k}\right\}_{k}$ converges strongly to $y_{\infty}$, the so-called limit profile defined (with $\hat{D}=0$ for ease of presentation) over $p=0,1, \ldots, \alpha-1$ by

$$
\begin{aligned}
x_{\infty}(p+1) & =\left(\hat{A}+\hat{B}_{0}\left(I-\hat{D}_{0}\right)^{-1} \hat{C}\right) x_{\infty}(p) \\
& +B u_{\infty}(p), \\
\hat{y}_{\infty}(p) & =\left(I-\hat{D}_{0}\right)^{-1} \hat{C} \hat{x}_{\infty}(p), \\
\hat{x}_{\infty}(0) & =\hat{d}_{\infty},
\end{aligned}
$$

where $\hat{d}_{\infty}$ is the strong limit of the state initial vector $\left(x_{k+1}(0), k \geq 0\right)$ sequence. In effect, this result states that if a process is asymptotically stable then its repetitive dynamics can, after a sufficiently large number of passes, be replaced by those of a 1D differential linear system. Note, however, that this property does not guarantee that the limit profile is stable as a $1 \mathrm{D}$ discrete linear system, i.e. $\mathrm{r}\left(\hat{A}+\hat{B}_{0}\left(I-\hat{D}_{0}\right)^{-1} \hat{C}\right)<1$, a point which is easily illustrated by the case when $\hat{A}=-0.5, \hat{B}=0, \hat{B}_{0}=0.5+\beta, \hat{C}=1$, $\hat{D}=0, \hat{D}_{0}=0$ and $\beta$ is a real scalar such that $|\beta| \geq 1$.

The reason why asymptotic stability does not guarantee a limit profile which is stable along the pass is due to the finite pass length. In particular, asymptotic stability is easily shown to be bounded-input bounded-output (BIBO) stability with respect to the finite and fixed pass length. Also in cases where this feature is not acceptable, the stronger concept of stability along the pass must be used. In effect, for the model (1), this requires that the BIBO stability property holds uniformly with respect to the pass length $\alpha$.

For the processes considered here, there are a wide range of stability along the pass tests but in this work it is the following LMI based condition that is the starting point for control law design in the ILC case.

Theorem 1: [5] A discrete linear repetitive process described by (2) is stable along the pass if there exist matrices $Y \succ 0$ and $Z \succ 0$ such that the following LMI holds

$$
\left[\begin{array}{ccc}
Y-Z & * & * \\
0 & -Z & * \\
\hat{A}_{1} Y & \hat{A}_{2} Y & -Y
\end{array}\right] \prec 0
$$


where

$$
\hat{A}_{1}=\left[\begin{array}{cc}
\hat{A} & \hat{B}_{0} \\
0 & 0
\end{array}\right], \hat{A}_{2}=\left[\begin{array}{cc}
0 & 0 \\
\hat{C} & \hat{D}_{0}
\end{array}\right] .
$$

We also make use of the following result.

Theorem 2: [6] For any $\mathcal{F}^{T} \mathcal{F} \leq I$ and a scalar $\epsilon>0$ the following holds

$$
\Sigma_{1} \mathcal{F} \Sigma_{2}+\Sigma_{2}^{T} \mathcal{F} \Sigma_{1}^{T} \leq \epsilon^{-1} \Sigma_{1} \Sigma_{1}^{T}+\epsilon \Sigma_{2}^{T} \Sigma_{2} .
$$

\section{ILC DESIGN}

Consider again (1). In many applications there will always be uncertainty associated with the plant dynamics and hence there is a need for robust design algorithms. Then to undertake robust ILC design in a repetitive process setting we consider the case when this is modeled as additive uncertainty in the state-space matrices of the model (1) of the form

$$
\begin{aligned}
& A=\tilde{A}+\Delta A, \\
& B=\tilde{B}+\Delta B,
\end{aligned}
$$

where $\tilde{A}$ and $\tilde{B}$ represent the nominal versions of $A$ and $B$ respectively and $\Delta A$ and $\Delta B$ are uncertainties that by assumption satisfy

$$
\begin{aligned}
\Delta A & =H_{1} F_{1} E_{1}, \\
\Delta B & =H_{2} F_{2} E_{2},
\end{aligned}
$$

where $F_{1}=F_{1}^{T}, F_{1}^{T} F_{1} \preceq I, F_{2}=F_{2}^{T}, F_{2}^{T} F_{2} \preceq I$ and $F_{1}=\mathbb{R}^{n \times n}, F_{2}=\mathbb{R}^{n \times n}, H_{1}, E_{1}, H_{2}, E_{2}$ are matrices of appropriate dimensions. Here $H_{1}, H_{2}, E_{1}$ and $E_{2}$ are assumed constant but $F_{1}$ and $F_{2}$ can vary both from trialto-trial and from point-to-point along the trial, but under the requirement that they are norm bounded. Here we aim to control the ILC dynamics by a linear control law which only uses plant output information.

Let $y_{\text {ref }}(p)$ denote the reference signal and $e_{k}(p)=$ $y_{\text {ref }}(p)-y_{k}(p)$ the error on trial $k$. Then it is easy to see that in this case

$$
\begin{aligned}
e_{k+1}(p)-e_{k}(p) & =C A\left(-x_{k+1}(p-1)+x_{k}(p-1)\right) \\
& +C B\left(-u_{k+1}(p-1)+u_{k}(p-1)\right)
\end{aligned}
$$

and introduce

$$
\begin{aligned}
\eta_{k+1}(p+1) & =x_{k+1}(p)-x_{k}(p), \\
\Delta u_{k+1}(p) & =u_{k+1}(p)-u_{k}(p) .
\end{aligned}
$$

Then in [7] it has been shown how to design the control law

$$
\Delta u_{k+1}(p)=K_{1} \eta_{k+1}(p+1)+K_{3} e_{k}(p+1),
$$

using an LMI setting to ensure stability along the trial. The only difficulty with this analysis is that in many cases the state vector $x_{k}(p)$ may not be available or, at best, only some of its entries are and hence, in general, an observer will be required. An alternative is to use a control law of the form

$$
\Delta u_{k+1}(p)=K_{1} \mu_{k+1}(p+1)+K_{2} \mu_{k+1}(p)+K_{3} e_{k}(p+1),
$$

where

$$
\mu_{k}(p)=y_{k}(p-1)-y_{k-1}(p-1)=C \eta_{k}(p) .
$$

Note that in comparison with (10) the vector variable $\eta_{k+1}(p+1)$ has been replaced by $\mu_{k+1}(p+1)$. The extra term in the control law considered here has been added as a means, if necessary, of compensating for the effects of replacing pure state vector information.

Now introduce

$$
\begin{aligned}
\tilde{\eta}_{k+1}(p+1) & =\widehat{A} \tilde{\eta}_{k+1}(p)+\widehat{B}_{0} e_{k}(p), \\
e_{k+1}(p) & =\widehat{C} \tilde{\eta}_{k+1}(p)+\widehat{D}_{0} e_{k}(p),
\end{aligned}
$$

where $\tilde{\eta}(p+1)=\left[\begin{array}{ll}\eta_{k+1}^{T}(p+1) & \eta_{k+1}^{T}(p)\end{array}\right]^{T}$ and

$$
\begin{aligned}
\widehat{A} & =\left[\begin{array}{c}
\tilde{A}+\Delta A+(\tilde{B}+\Delta B) K_{1} C \\
I
\end{array}\right. \\
\widehat{B}_{0} & =\left[\begin{array}{c}
(\tilde{B}+\Delta B) K_{2} C \\
(\tilde{B}+\Delta B) K_{3} \\
0
\end{array}\right], \\
\widehat{C} & =\left[\begin{array}{l}
-C A-C B K_{1} C \mid-C B K_{2} C
\end{array}\right], \\
\widehat{D}_{0} & =\left(I-C B K_{3}\right),
\end{aligned}
$$

which is of the form (2) and hence the repetitive process stability theory can be applied to this ILC control scheme. In particular, stability along the pass (or trial in the ILC setting) is equivalent to uniform bounded input bounded output stability (defined in terms of the norm on the underlying function space), i.e. independent of the trial length, and hence we can (potentially) achieve trial-trial error convergence with acceptable along the trial dynamics. (See also [8] where the same control law was developed for the case when no uncertainty is present in the process model.)

Note 1: A necessary condition for stability along the trial is $r\left(\widehat{D}_{0}\right)=r\left(I-C B K_{3}\right)<1$ which is the necessary and sufficient condition for asymptotic stability in this case. This last condition is precisely that obtained by applying 2D discrete linear systems stability theory to (13) as first proposed in [4] to ensure trial-to-trial error convergence only. The case where this could lead to unacceptable along the trial dynamics is illustrated by any example where the resulting limit profile is unstable as a standard linear system, i.e., $r(\widehat{A})<1$.

Now we have the following result.

Theorem 3: An ILC scheme described by (13) is stable along the trial if there exist matrices $Y \succ 0, Z \succ 0, N_{1}, N_{2}$, $N_{3}, P, Q$ and real scalars $\tilde{e}_{1}>0$ and $\tilde{e}_{2}>0$ such that the following LMI with linear constraints holds

$$
\begin{aligned}
& {\left[\begin{array}{cccccc}
Z-Y & 0 & \Omega_{31}^{T} & 0 & \Omega_{51}^{T} & \Omega_{61}^{T} \\
0 & -Z & \Omega_{32}^{T} & 0 & 0 & \Omega_{62}^{T} \\
\Omega_{31} & \Omega_{32} & \Omega_{33} & \Omega_{43}^{T} & \Omega_{53}^{T} & 0 \\
0 & 0 & \Omega_{43} & \Omega_{44} & 0 & 0 \\
\Omega_{51} & 0 & \Omega_{53} & 0 & \Omega_{55} & 0 \\
\Omega_{61} & \Omega_{62} & 0 & 0 & 0 & \Omega_{66}
\end{array}\right] \prec 0,} \\
& C Y_{1}=P C, C Y_{2}=Q C,
\end{aligned}
$$


where

$$
\begin{aligned}
& Y=\left[\begin{array}{ccc}
Y_{1} & 0 & 0 \\
0 & Y_{2} & 0 \\
0 & 0 & Y_{3}
\end{array}\right]
\end{aligned}
$$

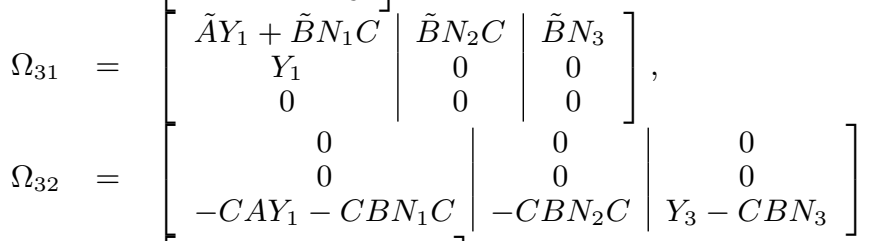

$$
\begin{aligned}
& \Omega_{33}=\left[\begin{array}{ccc}
-Y_{1} & 0 & 0 \\
0 & -Y_{2} & 0 \\
0 & 0 & -Y_{3}
\end{array}\right] \text {, } \\
& \Omega_{43}=\left[\begin{array}{ccc}
\tilde{e}_{2} H_{2}^{T} & 0 & 0 \\
0 & \tilde{e}_{2} H_{2}^{T} & 0 \\
0 & 0 & -\tilde{e}_{2} H_{2}^{T} C^{T}
\end{array}\right] \text {, } \\
& \Omega_{44}=\left[\begin{array}{ccc}
-\tilde{e}_{2} I & 0 & 0 \\
0 & -\tilde{e}_{2} I & 0 \\
0 & 0 & -\tilde{e}_{2} I
\end{array}\right] \\
& \Omega_{51}=\left[\begin{array}{ccc}
0 & 0 & 0 \\
0 & 0 & 0 \\
E_{2} N_{1} C & E_{2} N_{2} C & E_{2} N_{3}
\end{array}\right] \text {, } \\
& \Omega_{53}=\left[\begin{array}{ccc}
\tilde{e}_{1} H_{1}^{T} & 0 & 0 \\
0 & 0 & -\tilde{e}_{1} H_{1}^{T} C^{T} \\
0 & 0 & 0 \\
-\tilde{e}_{1} I & 0 & 0 \\
0 & -\tilde{e}_{1} I & 0 \\
0 & 0 & -\tilde{e}_{2} I
\end{array}\right] \\
& \Omega_{61}=\left[\begin{array}{ccc}
0 & 0 & 0 \\
E_{1} Y_{1} & 0 & 0 \\
0 & 0 & 0
\end{array}\right] \\
& \Omega_{62}=\left[\begin{array}{ccc}
E_{2} N_{1} C & E_{2} N_{2} C & E_{2} N_{3} \\
0 & 0 & 0 \\
E_{1} Y_{1} & 0 & 0 \\
-\tilde{e}_{2} I & 0 & 0 \\
0 & -\tilde{e}_{1} I & 0 \\
0 & 0 & -\tilde{e}_{1} I
\end{array}\right]
\end{aligned}
$$

If this LMI with equality constraints is feasible, control law matrices can be calculated using

$$
K_{1}=N_{1} P^{-1}, K_{2}=N_{2} Q^{-1}, K_{3}=N_{3} Y_{3}^{-1} \text {. }
$$

Proof: The first step is to interpret Theorem 1 in terms of the controlled dynamics and then use Theorem 2 in a manner that directly follows repetitive process analysis and the details can be found in [5].

The result of Theorem 3 provides infinitely many solutions for the control law matrices where in the example here these are scalars. To select these, we use the following objective function

$$
f\left(N_{2}, N_{3}, Y_{3}\right)=\sum N_{2} \cdot h_{1}-\sum N_{3} \cdot h_{2}+\sum Y_{3} \cdot h_{3},
$$

where $h_{k}, k=1,2,3$, are appropriately chosen constants and sums involved are performed for all elements of the appropriate matrices. Tweaking this function (e.g. by modifying $h_{k}$ ) allows us to obtain a set of control law matrices which satisfy (15) and this can also be used to tune performance.

To apply the control law, note that, after simple algebraic manipulations, we obtain

$$
\begin{aligned}
u_{k}(p)= & u_{k-1}(p)+K_{1}\left(y_{k}(p)-y_{k-1}(p)\right) \\
& +K_{2}\left(y_{k}(p-1)-y_{k-1}(p-1)\right) \\
& +K_{3}\left(y_{\text {ref }}(p+1)-y_{k-1}(p+1)\right) .
\end{aligned}
$$

Here the last term is phase advance on the previous trial error, where in ILC such a term is well known in simple structure algorithms. Such an advance appears in the discrete-time implementation of the derivative ILC algorithm, where it was used to extend the applicability of the original ILC algorithm [1]. Since then, a variable advance has been considered [9] and found to lead to accurate training (or learning) in practice on a range of systems [10]. The second and third terms are proportional in nature acting on the error between the current and previous pass trials at $p$ and $p-1$ respectively. Whilst use of current trial data has appeared in many approaches to manipulate the plant dynamics along the trial [11] and has been found to increase initial tracking and disturbance rejection [12], the coupling of previous and current trial data is a novel addition to this class of updates.

Implementation of this control law does not require a state observer (in repetitive process terms $y$ is the process output and hence available for measurement by definition) but does assume in this first work that the level of noise and other disturbances on the measurements is negligible. Note also that this control law design method covers both the multipleinput multiple-output (MIMO) and single-input single-output (SISO) cases.

\section{EXPERIMENTAL VERIFICATION}

The new ILC control law design algorithms developed in this paper have been experimentally validated using a multiaxis gantry robot, see Fig. 1, previously used for testing and comparing the performance of other ILC algorithms, see, for example, [12] and this section gives (some of) the results obtained together with supporting discussion. Each axis of the gantry robot is modeled from frequency response data where, since the axes are orthogonal, it is assumed that there is minimal interaction between them. Consider the $X$-axis (the one parallel to the conveyor in Fig. 1), for which frequency response tests (using the Bode gain and phase plots in Figure 2) result in a 7th order continuous time transfer-function given in [12] as an adequate model of the dynamics to use for control law design (this reference also gives the models for the $Y$ and $Z$ axes). The transferfunction is discretized with a sampling time of $T_{s}=0.05$ seconds to develop a discrete linear state-space model. The required reference trajectory is designed to simulate a "pick and place" process of duration 2 seconds, and the signal is used in all algorithm tests in order to make all results comparable. The $X$-axis component of the $3 \mathrm{D}$ reference trajectory is shown in Figure 3 (and for brevity we only consider this axis from this point onwards).

We also consider uncertainty defined by the following matrices, which move at least one eigenvalue of the matrix $A$ outside the unit circle and hence a necessary condition for stability along the trial is violated. 


$$
H_{1}=\left[\begin{array}{cccc}
0.0019 & 0.0042 & 0.0001 & 0.0016 \\
0.0038 & 0.0047 & 0.0045 & 0.0045 \\
0.0042 & 0.0008 & 0.0048 & 0.0047 \\
0.0016 & 0.0009 & 0.0001 & 0.0046 \\
0.0011 & 0.0010 & 0.0027 & 0.0006 \\
0.0050 & 0.0049 & 0.0041 & 0.0000 \\
0.0004 & 0.0005 & 0.0046 & 0.0010
\end{array}\right.
$$

$\left.\begin{array}{lll}0.0046 & 0.0003 & 0.0011 \\ 0.0005 & 0.0003 & 0.0017 \\ 0.0026 & 0.0033 & 0.0048 \\ 0.0018 & 0.0050 & 0.0021 \\ 0.0000 & 0.0010 & 0.0032 \\ 0.0018 & 0.0020 & 0.0013 \\ 0.0034 & 0.0047 & 0.0024\end{array}\right]$

\begin{tabular}{|c|c|c|c|c|}
\hline 0.0037 & 0.0031 & 0.0032 & & \\
\hline 0.0014 & 0.0001 & 0.0033 & \multirow{6}{*}{, } & \\
\hline 0.0020 & 0.0001 & 0.0028 & & \\
\hline 0.0028 & 0.0019 & 0.0045 & & \\
\hline 0.0018 & 0.0047 & 0.0039 & & \\
\hline 0.0030 & 0.0032 & 0.0047 & & \\
\hline 0.0037 & 0.0039 & 0.0048 & & \\
\hline \multirow{7}{*}{$E_{1}=$} & 0.061 & 0.024 & 0.092 & 0.019 \\
\hline & 0.006 & 0.062 & 0.032 & 0.039 \\
\hline & 0.006 & 0.031 & 0.042 & 0.099 \\
\hline & 0.030 & 0.074 & 0.055 & 0.089 \\
\hline & 0.041 & 0.100 & 0.028 & 0.088 \\
\hline & 0.027 & 0.000 & 0.094 & 0.098 \\
\hline & 0.015 & 0.062 & 0.082 & 0.006 \\
\hline
\end{tabular}

$H_{2}=\left[\begin{array}{llll}0.0050 & 0.0003 & 0.0021 & 0.0009 \\ 0.0004 & 0.0021 & 0.0017 & 0.0010 \\ 0.0047 & 0.0044 & 0.0017 & 0.0035 \\ 0.0047 & 0.0043 & 0.0017 & 0.0035 \\ 0.0035 & 0.0004 & 0.0040 & 0.0015 \\ 0.0036 & 0.0046 & 0.0010 & 0.0002 \\ 0.0031 & 0.0010 & 0.0006 & 0.0027\end{array}\right.$

$\left.\begin{array}{lll}0.0342 & 0.049 & 0.027 \\ 0.0794 & 0.095 & 0.043 \\ 0.0585 & 0.092 & 0.030 \\ 0.0304 & 0.019 & 0.036 \\ 0.0336 & 0.025 & 0.042 \\ 0.0946 & 0.035 & 0.085 \\ 0.0835 & 0.079 & 0.079\end{array}\right], E_{2}=\left[\begin{array}{l}0.024 \\ 0.093 \\ 0.039 \\ 0.060 \\ 0.014 \\ 0.050 \\ 0.060\end{array}\right]$,

and both $F_{1}$ and $F_{2}$ are taken as a $7 \times 7$ diagonal matrix with each non-zero entry set at 0.99 . For this data $r(A)=1.0017$ and applying Theorem 3 (with $h_{1}=0.001, h_{2}=-1, h_{3}=$ 25 in (18)) gives

$$
K_{1}=-121.53, K_{2}=-13.88, K_{3}=57.46 .
$$

This control law was applied to the robot where hardware constraints necessitated the use of a multi-sampling approach (for a similar technique see, for example, [13]). The basic idea of a multi-sampling algorithm is that we sample the system with relatively large sampling period $T_{s, \max }$ and replace a single low-frequency (with sampling frequency $f_{s . \min }=\frac{1}{T_{s, \max }}$ ) controller by $\hat{n}$ low-frequency controllers working independently on different parts of the system frequency response. This task is accomplished by simply dividing $T_{s, \max }$ into $\hat{n}$ equal parts, i.e. $T_{s, c}=\frac{T_{s, \max }}{\hat{n}}$. Each controller $k(k=0,1, \ldots, \hat{n}-1)$ is designed for a plant model sampled with period $T_{s, \max }$ and works also with this period but starts from $k \cdot T_{s, c}, \quad k=0,1, \ldots, \hat{n}-1$. These $\hat{n}$ controllers can be regarded as one super-controller operating with sampling period $T_{s, c}$.

Figure 4 shows the progression of the input, output and error as the trials are completed and Fig 5 the mean squared error against trial number. These show that the objectives of trial-to-trial error convergence and along the trial performance can both be well controlled and further refinement to, for example, place stronger emphasis on the mean square error reduction is possible.

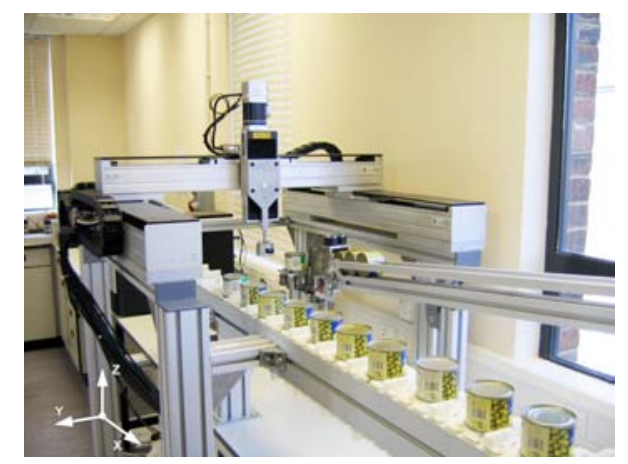

Fig. 1. The gantry robot with the axes marked.
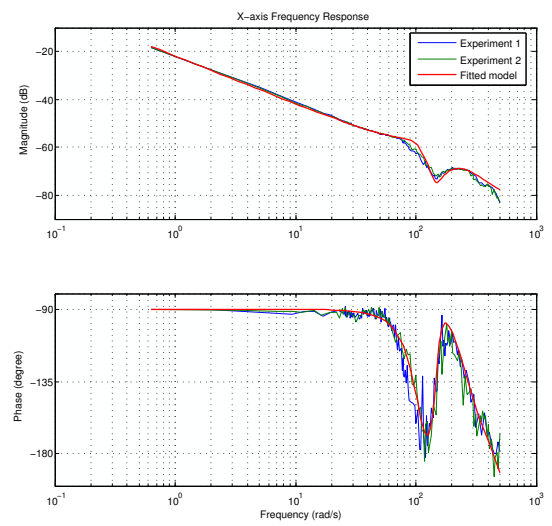

Fig. 2. Bode gain and phase plots of the $X$-axis frequency response.

\section{CONCLUSIONS}

This paper has shown how 2D systems theory, in the form of stability analysis for linear repetitive processes can be used to design ILC laws in the presence of uncertainty. The resulting design has been experimentally verified for a case where in the absence of control action the uncertainty results in an unstable along the trial process. Moreover, the final control law has a well defined physical structure and can 

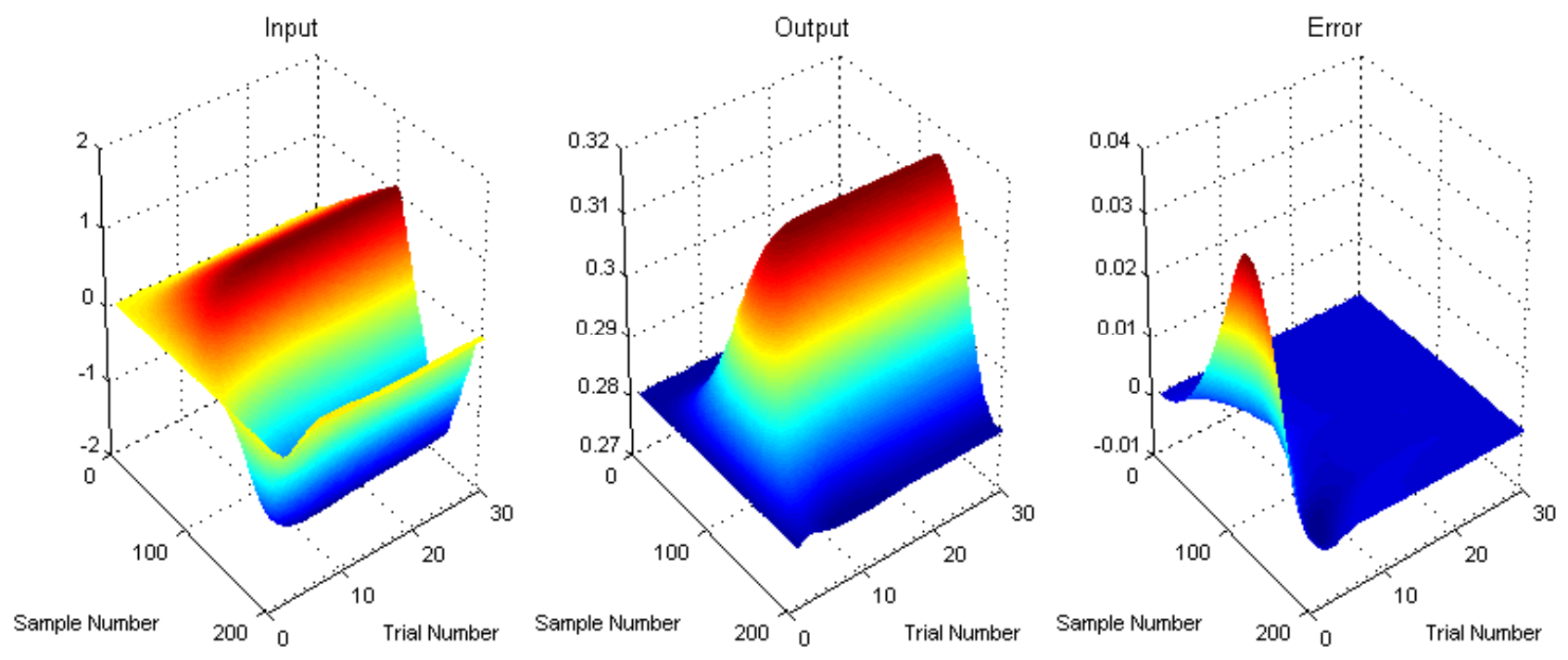

Fig. 4. Experimentally measured input (left), output (center) and error (right) progression with trial number.

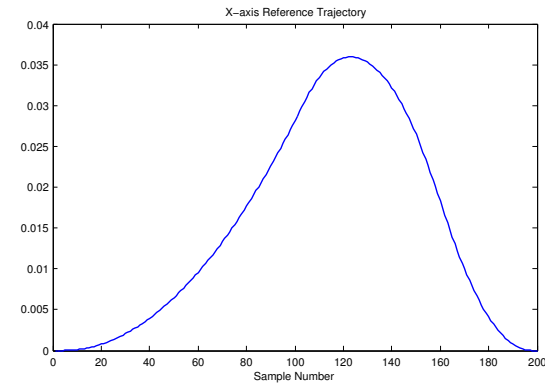

Fig. 3. The $X$-axis reference trajectory.

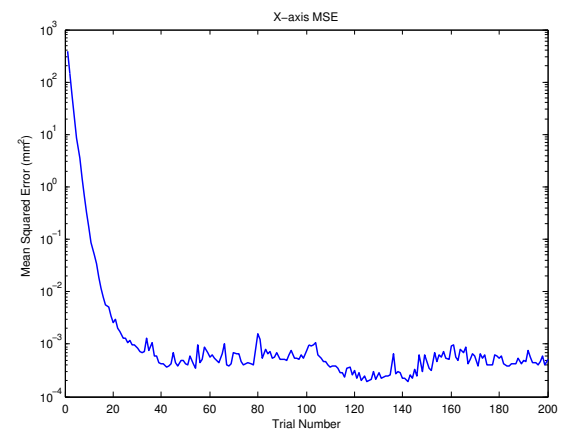

Fig. 5. Experimentally measured mean square error plotted against trial number.

be implemented without the need for state vector estimation. These results are a significant advance in terms of ILC design using a 2D systems setting. Of course, there are many other possible ways of studying the robustness problem for ILC and the experimental results, in particular, also provide a basis for comparison against alternatives.

\section{REFERENCES}

[1] S. Arimoto, S. Kawamura, and F. Miyazaki, "Bettering operations of robots by learning," Journal of Robotic Systems, vol. 1, no. 2, pp.
123-140, 1984.

[2] D. A. Bristow, M. Tharayil, and A. G. Alleyne, "A survey of iterative learning control," IEEE Control Systems Magazine, vol. 26, no. 3, pp. 96-114, 2006.

[3] H.-S. Ahn, Y. Chen, and K. L. Moore, "Iterative learning control: brief survey and categorization," IEEE Transactions on Systems, Man and Cybernetics, Part C, vol. 37, no. 6, pp. 1109-1121, 2007.

[4] J. E. Kurek and M. B. Zaremba, "Iterative learning control synthesis based on 2-D system theory," IEEE Transactions on Automatic Control, vol. 38, no. 1, pp. 121-125, 1993.

[5] E. Rogers, K. Galkowski, and D. H. Owens, Control Systems Theory and Applications for Linear Repetitive Processes, ser. Lecture Notes in Control and Information Sciences. Springer Verlag, 2007, vol. 349.

[6] P. P. Khargonekar, I. R. Petersen, and K. Zhou, "Robust stabilization of uncertain linear systems: quadratic stabilizability and $H_{\infty}$ control theory," IEEE Transactions on Automatic Control, vol. 35, no. 3, pp. 356-361, 1990.

[7] L. Hladowski, K. Galkowski, Z. Cai, E. Rogers, C. T. Freeman, and P. L. Lewin, "A 2D systems approach to iterative learning control with experimental validation," in Proc. of the 17th IFAC World Congress, Soeul, Korea, July 2008, pp. 2832-2837.

[8] L. Hladowski, Z. Cai, K. Galkowski, E. Rogers, C. Freeman, and P. L. Lewin, "Using 2D systems theory to design output signal based iterative learning control laws with experimental verification," in Proceedings of the 47th IEEE Conference on Decision and Control, December 2008, pp. 3026-3031.

[9] Y. Wang and R. W. Longman, "Use of non-causal digital signal processing in learning and repetitive control," Advances in the Astronautical Sciences, vol. 90, no. 1, pp. 649-668, 1996.

[10] J. Wallen, M. Norrlof, and S. Gunnarsson, "Arm-side evaluation of ILC applied to a six-degrees-of-freedom industrial robot," in Proceedings of 17th IFAC World Congress 2008, Seoul, Korea, July 2008, pp. $13450-$ 13455.

[11] M. Norrlof and S. Gunnarsson, "A frequency domain analysis of a second order iterative learning control algorithm," in Proceedings of the 38th IEEE Conference on Decision and Control, Phoenix, Arizona, USA, December 1999, pp. 1587-1592.

[12] J. D. Ratcliffe, J. J. Hatonen, P. L. Lewin, E. Rogers, T. J. Harte, and D. H. Owens, "P-type iterative learning control for systems that contain resonance," International Journal of Adaptive Control and Signal Processing, vol. 19, no. 10, pp. 769-796, 2005.

[13] B. Zhang, D. Wang, K. K. Zhou, Y. Ye, and Y. Wang, "Cyclic pseudodownsampled iterative learning control for high performance tracking," Control Engineering Practice, vol. 17, no. 8, pp. 957-965, 2008. 